\title{
GroE is vital for cell-wall synthesis
}

Chaperone proteins help other proteins $\checkmark$ to fold. GroEL, the Escherichia coli form of the ubiquitous Cpn60 chaperonins, has a multimeric barrel-shaped structure with a central cavity, within which almost any protein can fold in vitro ${ }^{1}$. But what does GroE (GroEL plus its co-chaperone GroES) fold in the cell? Why is it needed for cell survival $^{2}$ ? We report here the first definite identification of an essential, GroE-dependent E. coli protein, dihydropicolinate synthase (DapA), without which cell-wall synthesis fails.

Although GroE is abundant, calculation ${ }^{3}$ suggests there is enough in a cell to interact with only about $10 \%$ of newly synthesized proteins. While several hundred different species of newly synthesized protein transiently associate with GroE, they comprise fewer than $20 \%$ of possible types ${ }^{4}$, only a minority of which are likely to be essential.

We find that cells deprived of GroE lyse because they fail to make cell walls. Lysis is due to a lack of the cell-wall precursor diaminopimelic acid (DAP), which fails to be made because the level of DapA - the first enzyme in the DAP-synthesis pathway - is greatly reduced in GroE-deficient cells.

When GroE levels were reduced ${ }^{5}$ or a mutant form of the protein inactivated by temperature shift ${ }^{6}$, changes in the levels of many proteins were noted, but no particular protein was identified as both GroEdependent and essential for cell survival. By replacing the native groE chromosomal promoter region with the $\operatorname{araC}$ gene and $\operatorname{ara}_{B A D}$ promoter (Fig. 1a), we created a strain dependent on added arabinose for continued viability and GroE production, and were able to use it to determine the cause of death as GroE is depleted.

Following arabinose removal, GroE levels halve with each generation (Fig. 1b). Growth remains exponential for about 2 hours (Fig. 2a; GroE level will be reduced to $2-3 \%$ of normal by this time) and then continues at a decreasing rate for another 1.5 hours; the cells then abruptly lyse. That cells can continue to grow exponentially with greatly reduced levels of GroE suggests either that any essential GroE-dependent proteins are present in considerable excess, or that they can effectively co-opt any GroE available ${ }^{3}$.

During GroE depletion in osmotically protected medium, the cells are converted to spherical protoplasts, indicating a defect in synthesis or maintenance of the peptidoglycan cell wall. We followed peptidoglycan synthesis by measuring the rate of incorporation of $\left[{ }^{3} \mathrm{H}\right] \mathrm{DAP}$, a specific precursor, into peptidoglycan ${ }^{7}$. Uptake was greatly enhanced in GroE- depleted cells, suggest-

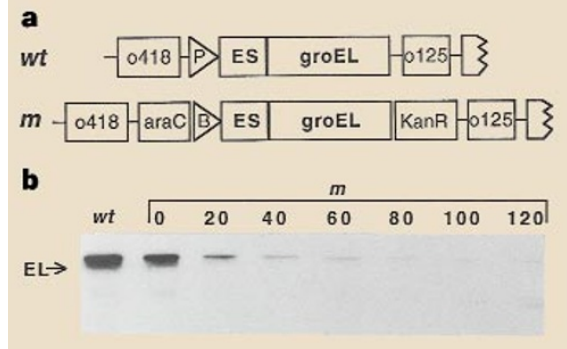

Figure 1 Depletion of GroE in E. coli. a, Genetic organization of the groESL region. wt, the native groE operon and surrounding open-reading frames. $\mathrm{P}$ represents the native groE promoters ( $\sigma 32$ and $\sigma 70$ ) and $E S$ the groES gene. $\mathrm{m}$, The groE region in the 'depletion mutant' strain. The araC gene and the arabinose-dependent $P_{B A D}$ promoter $(B)$ replace the native promoter; a kanamycin-resistance cassette is downstream of groE. b, GroEl depletion after arabinose removal. Mutant cells grown in L-broth at $37^{\circ} \mathrm{C}$ with $0.1 \%$ arabinose were centrifuged, washed, diluted into broth with $0.2 \%$ glucose (time zero) and growth followed at $37^{\circ} \mathrm{C}$. Extracts of aliquots taken between 0 and 120 minutes were electrophoresed, electroblotted onto nitrocellulose, and GroEL (EL) was detected by chemiluminescence using polyclonal antiserum. An extract of the parental wildtype strain, MG 1655, is included (wt). The mutant strain grown with $0.1 \%$ arabinose produces $\sim 80 \%$ as much GroEL as does MG 1655 .

ing that these cells might be DAP-starved.

In confirmation of this, we found that addition of DAP to the growth medium (Fig. 2a) prevents cell lysis and permits growth to continue for up to 6 hours. Furthermore, although addition of DAP to solid medium does not support colony formation - confirming that GroE is essential for processes other than cell wall synthesis - when DAP is present, colony formation requires less arabinose than is normally the case. This suggests that at least a part of the cellular complement of GroE is dedicated to ensuring that DAP is available, and that GroE is likely to have a role in maintaining the levels of an enzyme or enzymes involved in DAP synthesis.

DAP is synthesized from aspartic semialdehyde in six steps ${ }^{8}$. Deficiency of any of the enzymes DapA-E causes cell lysis ${ }^{9}$. We reasoned that if a critical enzyme were to be synthesized in excess before GroE depletion, lysis would be delayed. We cloned $\operatorname{dap} A, B$, $D$ and $E$ separately into pBR325 to achieve overproduction of the encoded enzymes. The presence of the dapA, but not of the other dap plasmids, delays lysis by several hours (Fig. 2a), suggesting that levels of DapA may be influenced by GroE. Immediately before lysis, the level of DapA in the depleted cells is reduced to $16 \%$ of normal (Fig. 2b). The remaining protein is soluble

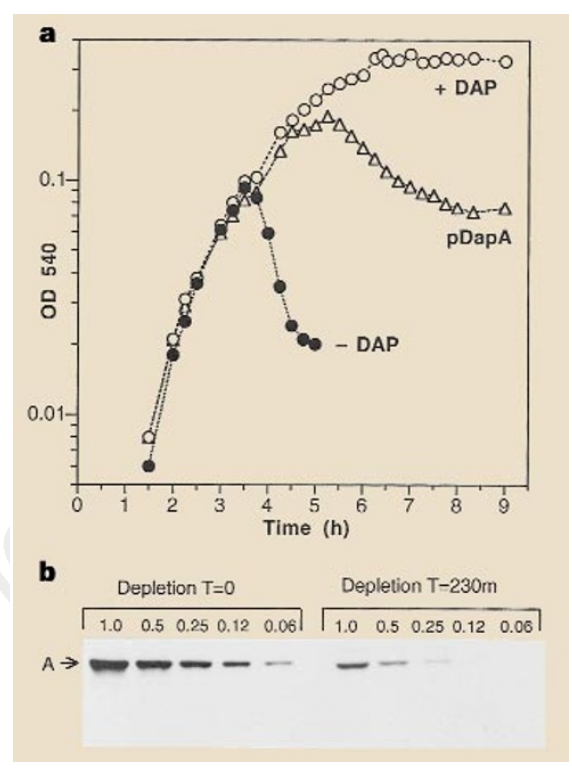

Figure 2 DapA loss in GroE-depleted cells. a, Cells depleted of GroE lyse (-DAP); addition of diaminopimelic acid (+DAP) prevents lysis. Lysis is delayed in cells in which DapA is overproduced (pDapA). The mutant was grown as for Fig. 1b, with or without DAP $\left(50 \mu \mathrm{g} \mathrm{ml}^{-1}\right)$. dapA was amplified by polymerase chain reaction and cloned into pBR325 (pDapA). pDapA was then transformed into the mutant. b, Levels of DapA decrease with GroE depletion. Mutant cells were grown and extracts prepared at the indicated times, as for Fig. 1b. Samples containing equal amounts of protein were serially diluted as indicated. Western blots were prepared using anti-DapA antiserum (gifts from G. Galili and C. Falco).

and stable. In contrast, the levels of several other essential proteins examined do not decrease during GroE depletion.

Thus we see that levels of an essential protein, DapA, are dependent on GroE. We suggest that decreased stability of nascent DapA, as a consequence of failure of GroEassisted folding, is the most likely reason for DapA deficiency.

Neil McLennan, Millicent Masters Institute of Cell and Molecular Biology, Edinburgh University, Kings Buildings,

Mayfield Road, Edinburgh EH9 3JR, UK

e-mail:M.Masters@ed.ac.uk

\footnotetext{
1. Martin, J. \& Hartl, F. U. Curr. Opin. Struct. Biol. 7, 41-52 (1997).

2. Fayet, O., Ziegelhoffer, T. \& Georgopolous, C. J. Bacteriol. 171, 1379-1385 (1989).

3. Lorimer, G. H. FASEB J. 10, 5-9 (1996).

4. Ewalt, K. L. et. al. Cell 90, 491-500 (1997).

5. Kanemori, M., Mori, H. \& Yura, T. J. Bacteriol. 176, 4235-4242 (1994).

6. Horwich, A. L. et al. Cell 74, 909-917 (1993).

7. Höltje, J. V. et al. J. Bacteriol. 124, 1067-1076 (1975).

8. Cohen, G. N. in Amino Acids: Biosynthesis and Genetic Regulation (eds Herrmann, K. M. \& Somerville, R. L.) 147-171 (Addison-Wesley, Reading, MA., 1983).

9. Patte, J.-C. in Escherichia coli and Salmonella: Cellular and Molecular Biology, 2nd edn (ed. Neidhardt, F. C.) 528-541 (ASM, Washington DC, 1996).
} 